\title{
TEMA 13-2016: Intoxicación aguda por Ácido AcetilSalicílico. Parte 1: farmacología y fisiopatología
}

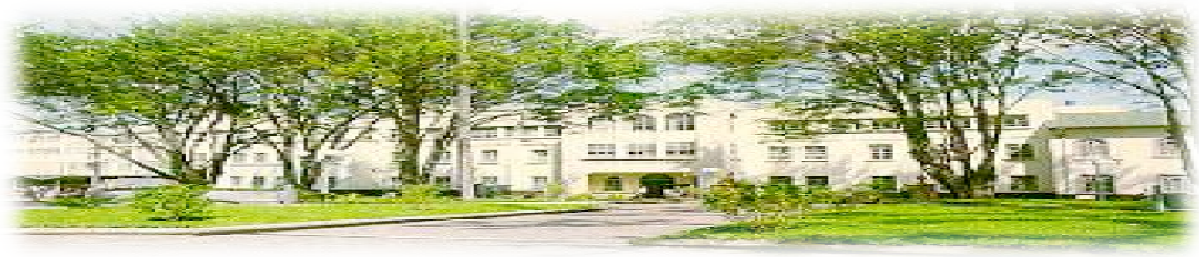

Hospital San Juan de Dios, San José, Costa Rica. Fundado en 1845

Recibido:05/01/2016

Aceptado:10/01/2016

Rolf Chaverri Jara ${ }^{1}$ Isabel Arce Jiménez ${ }^{2}$ Adriana Valverde Zúñiga ${ }^{3}$

${ }^{1}$ Médico especialista en Medicina de Emergencias, Hospital San Juan de Dios. Académico de la Escuela de Medicina de la Universidad de Costa Rica.

${ }^{2}$ Médico General. Universidad de Costa Rica. Correo electrónico: dra.arce@gmail.com

${ }^{3}$ Médico General. Universidad de Costa Rica. Correo electrónico: adri0719@hotmail.es

\section{RESUMEN}

El ácido acetil salicílicoes el analgésico, antipirético y antiinflamatorio de mayor uso en el mundo. La intoxicación aguda con salicilatos es entonces un cuadro frecuente, no obstante, a menudoel paciente no es adecuadamente reconocido o manejado. Esta revisión consta de dos partes, una base de la farmacología y fisiopatología del salicilismo agudo y un segundo artículo enfocado en el abordaje y detalles del manejo clínico. El conocimiento de la farmacocinética y farmacodinamia es vital para comprender el comportamiento clínico y la variabilidad entre pacientes. Asimismo, el entendimiento del proceso fisiopatológico tras la intoxicación asegura al médico la posibilidad de un abordaje integral. El objetivo de este artículo es discutir de forma exhaustiva pero práctica aspectos que faciliten al médico un manejo razonado del paciente.

\section{PALABRAS CLAVE}

Salicilismo, ácido acetil salicílico, aspirina, intoxicación, farmacodinamia, farmacocinética.

\section{ABSTRACT}

Acetylsalicylic acid (ASA) is the most commonly used analgesic, antipyretic and antiinflammatory drug worldwide. Acute intoxication is therefore frequent, nonetheless, patients are often not properly recognized or managed. This review consist of two parts, the first paper discusses the pharmacology and pathophysiology 
of acute salicylism and the second article deals with workup and treatment. Knowledge ofASA pharmacokinetics and pharmacodynamics is vital to understand the clinical presentation and variability. Likewise, comprehension of the pathophysiological mechanism of toxicity ensures an integral approach. The objective of this article is to discuss in an exhaustive and concrete manner the fundamentals that facilitate a reasonable patient management.

\section{KEY WORDS}

Salicylism, acetylsalicylic acid, aspirin, poisoning, pharmacodynamics, pharmacokinetics.

\section{INTRODUCCIÓN}

Los salicilatos, químicos derivados del ácido salicílico, han sido usados desde la edad antigua. Hipócrates, Galeno y los herboristas medievales los utilizaban por sus propiedades analgésicas y antipiréticas $^{(1)}$. Actualmente, a pesar del desarrollo de numerosos compuestos farmacológicos, el ácido acetilsalicílico (AAS o aspirina) continúa siendo el analgésico, antipirético y antiinflamatorio de mayor consumo en el mundo ${ }^{(2)}$. La aspirina se encuentra en cientos de preparaciones de fácil acceso, por lo que su potencial abuso y toxicidad grave son altos y posiblemente subestimados ${ }^{(3)}$.

La intoxicación por AAS es común en los servicios de emergencias, no obstante, en ocasiones no es oportunamente reconocida ${ }^{(4)}$.Con la finalidad de un diagnóstico temprano, el médico debe dominar el espectro de presentaciones clínicas que puede tener esta intoxicación. El entendimiento de la fisiopatología y alteraciones metabólicas que acompañan al cuadro es fundamental para brindar un manejo adecuado, prevenir complicaciones y disminuir la mortalidad.

\section{DISCUSIÓN}

\section{Principios farmacológicos}

\section{Grupo farmacológico}

Los AntiInflamatorios No Esteroideos (AINEs) son un grupo farmacológico de químicos hete- rogéneos, se asocian esencialmente por sus propiedades terapéuticas similares. Por su estructura, los AINEs se dividen en dos familias: ácidos carboxílicos y ácidos enólicos ${ }^{(5)}$.El primer subgrupo, se puede subdividir al menos en otras 7 categorías, una de las cuales es la de los derivados del ácido salicílico, dentro de la cual se encuentra el $\mathrm{AAS}^{(5)}$.También se pueden agrupar según su acción específica o no específica sobre las isoenzimas COX-1 y COX-2.

El ácido salicílico es tan irritante que solo puede usarse tópicamente, es por esto que se han desarrollado derivados para su uso sistémico ${ }^{(2)}$.El AAS es un éster del ácido acético, en el cual se realiza una sustitución acetil en el grupo hidroxilo (Figura 1).

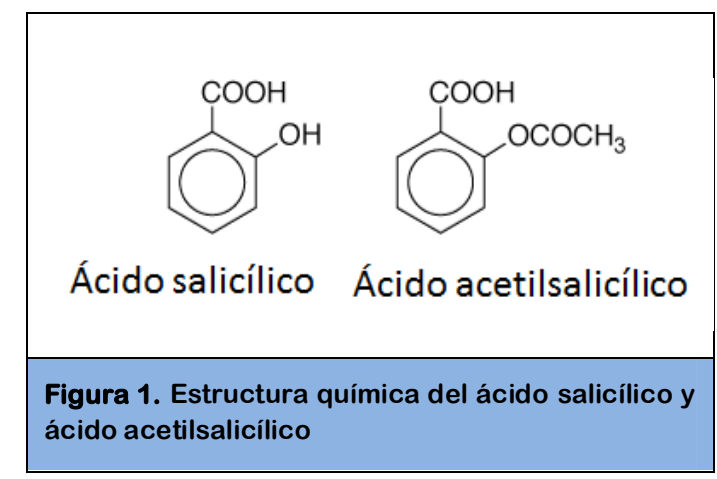

\section{Farmacocinética}

\section{Absorción}

La velocidad de absorción del AAS está determinada por múltiples factores. La forma farmacéutica es fundamental,aunque indudablemente, casi la totalidad de casos de intoxicación se dan por la vía oral ${ }^{(1)}$.Para las distintas formas farmacéuticas son determinantes la dosis, tasa de desintegración-disolución, el $\mathrm{pH}$ de la superficie mucosa y tiempo de vaciado gástrico ${ }^{(2)}$.A dosis terapéuticas, el AAS es rápidamente absorbido, a los 15-30 minutos de su ingesta se pueden detectar niveles en sangre y las concentraciones séricas máximas se alcanzan en 1-2 horas ${ }^{(2)}$.

Caso contrario, en sobredosis, las concentraciones pico pueden ser alcanzadas en 18-24 o hasta 60 horas $^{(3)}$. Se ha propuesto que la sobredosis de AAS puede causar prolongación del tiempo de vaciamiento gástrico y espasmo del esfinter pilórico, consecuentemente retrasando el paso y absorción en intestino delgado ${ }^{(1)}$.A la vez,la 
absorción se puede retrasar por la coingestión de agentes que disminuyan el vaciamiento gástrico y motilidad intestinal ${ }^{(1)}$.La absorción del AAS se ve modificada por la presencia de comida, la cual retrasa el proceso ${ }^{(4)}$. Dentro otros posibles determinantes de una absorción retardada, se menciona:anticolinérgicos, narcóticos, bloqueadores gangliónicos, hidróxido de aluminio, dolor, migraña, isoniazida, úlceras pépticas, íleo paralítico, abdomen agudo, trauma, infarto al miocar$\operatorname{dio}^{(16)}$.

Una distinción se describe con las tabletas de cubierta entérica, las cuales pueden crear bezoares que prolongan el tiempo de absorcióny se comportan como un medicamento de liberación sostenida $^{(4)}$. En este caso, los niveles séricos pueden encontrarse elevados de 60-70 horas posterior a la ingestión y no pueden ser utilizados como guía pronostica ${ }^{(1,17)}$. La ruptura de un bezoar suele ser catastrófica,especialmente si el mismo contiene también acetaminofén.

El AAS es un ácido débil ( $\mathrm{pKa}=3,5)$, en el ambiente de acidez estomacal, su equilibrio ácidobase se desplaza hacia su forma no ionizada. Por sus características eléctricas, puede atravesar pasivamente la membrana celular lipídica en la mucosa gastrointestinal, alcanzar el nivel intracelular y pasar hacia la circulación sistémica. Conforme aumenta el $\mathrm{pH}$ en intestino delgado, este salicilato se encontrará más ionizado ${ }^{(2)}$.No obstante, el medio más alcalino favorece la solubilidad y por tanto la disolución de tabletas ${ }^{(1 ; 2)}$. Lo anterior, sumado a la mayor superficie de absorción en el intestino delgado, determina que este sea el sitio de mayor absorción ${ }^{(1)}$.

\section{Distribución}

El salicilato se distribuye a los tejidos y fluidos transcelularespor un proceso pasivo ${ }^{(2,6)}$.El volumen de distribución (VD)depende de las concentraciones séricas del fármaco, de los niveles de albúmina y de la competencia con otras drogas por los sitios de unión a proteínas ${ }^{(7)}$.El VD a dosis terapéuticas en sujetos sanos es de $\sim 0,2$ $\mathrm{mL} / \mathrm{kg}^{(2,6)}$.A dosis mayores, aumenta por la saturación de los sitios de unión a proteínas ${ }^{(2)}$.El VD puede verse aumentado en la acidemia, la cual aumenta la internalización celular de la droga ${ }^{(7,8)}$.

La unión a proteínas es dosis dependiente y no lineal ${ }^{(9)}$. La proporción total de unión, sobre todo a la albúmina, disminuye a medida que aumenta la dosis ${ }^{(2)}$.A concentraciones bajas de salicilato $(<100 \mathrm{ug} / \mathrm{mL}) \sim 90 \%$ se une a albúmina, en concentraciones más altas $(>400 \mathrm{ug} / \mathrm{mL})$ sólo $\sim 75 \%$ se encuentra unido $^{(9)}$. En pacientes hipoalbuminémicos, un nivel desproporcionado de salicilato libre se encontrará en plasma ${ }^{(2)}$.Los salicilatos compiten por su sitio de unión a las proteínas plasmáticas con otros fármacos como la tiroxina, triyodotironina, penicilina, fenitoína, bilirrubina, ácido úrico y otros AINEs como el naproxeno ${ }^{(2)}$. En un menor grado, el AAS acetila la albúmina y puede cambiar el sitio de unión de otras drogas a la albúmina ${ }^{(2)}$.

Las concentraciones más altas de salicilato se encuentran en el plasma, hígado, corteza renal, corazón y pulmones ${ }^{(9)}$. Los salicilatos son transportados activamente por un sistema saturable de baja capacidad fuera del líquido cefalorraquídeo a través del plexo coroideo y fácilmente cruzan la barrera placentaria ${ }^{(2)}$. Dada la $\mathrm{pKa}$, a pH fisiológico casi todas las moléculas están ionizadas.Si el pH sistémico disminuye, se produce un equilibrio hacia una mayor proporción de moléculas no ionizadas, que pueden cruzar las membranas plasmáticas, como la barrera hematoencefálica $^{(3)}$.

\section{Metabolismo}

El AAS es absorbido principalmente como tal, pero una porción es hidrolizada por esterasasen la mucosa gastrointestinal y entra a la circulación como ácido salicílico ${ }^{(2)}$.Rápidamente, el AAS restante es hidrolizado en plasma, hígado y eritrocitos y solo una pequeña fracción se mantiene en la forma acetilada ${ }^{(3)}$.Las esterasas del AAS son dos enzimas plasmáticas, la "enzima C" similar a la colinesterasa plasmática y la "enzima A", una albúmina-esterasa de menor activi$\mathrm{dad}^{(9)}$.Este es un proceso de primer orden, con una vida media de 15-20 minutos ${ }^{(1)}$.

La biotransformación de los salicilatos se da en muchos tejidos, particularmente en el hígado, a nivel de retículo endoplásmico y mitocondria ${ }^{(2)}$.A dosis terapéuticas, el ácido salicílico sufre una biotransformación hepática, en un proceso de primer orden $^{(1)}$.Se describen tres reacciones principales (Figura 2$)^{(1)}$ : 
1. Glucoronización. Produce glucorónido fenólico salicílico y glucorónidoacíl salicílico.

2. Oxidación. Produce ácido gentísico.

3. Oxidación microsomal o conjugación con glicina. Produce ácido salicilúrico $(75 \%)$

Las vías metabólicas principales (ácido salicilúrico y glucorónido fenólico salicílico) se saturan cuando las concentraciones séricas de salicilato exceden los 20-30 mg/dL ${ }^{(1)}$.En este escenario, la biotransformación se convierte de un proceso dosis-dependiente de primer orden a un proceso de orden cero $^{(1)}$. En adelante, una cantidad fija de AAS se metabolizará por unidad de tiempo, independientemente de la dosis, favoreciendo la toxicidad $^{(1)}$.

Pequeños aumentos en la dosis pueden producir un aumento significativo de la concentración plasmática $^{(1)}$.El metabolismo hepático tiene una gran variabilidad inter-sujeto, debido a la contribución de las distintas vías metabólicas ${ }^{(2)}$.Las mujeres usualmente exhiben mayores concentraciones plasmáticas, posiblemente por una menor actividad esterasa intrínseca y diferencias de género en el metabolismo hepático ${ }^{(2)}$.

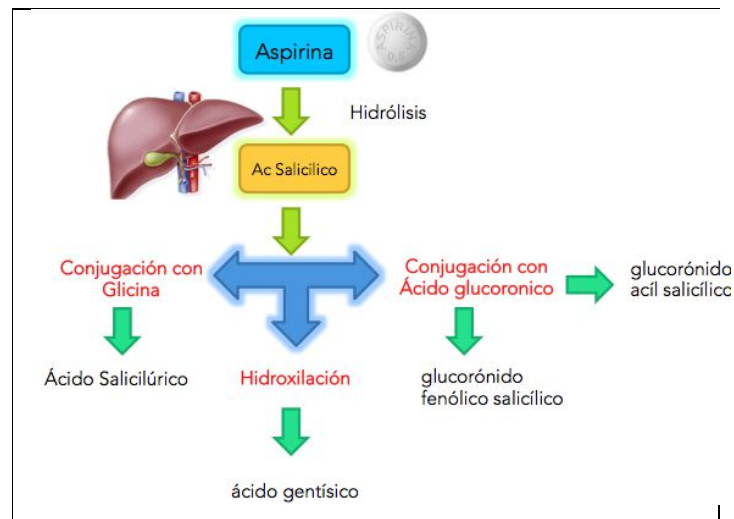

Figura 2. Metabolismo del AAS.

\section{Eliminación}

El ácido salicílico y sus metabolitos se eliminan principalmente por vía renal y su vida media $\left(\mathrm{t}^{1} / 2\right)$ varía dependiendo de la concentración plasmática y $\mathrm{pH}$ urinario ${ }^{(7)}$.Aproximadamente un $75 \%$ es eliminado como ácido salicilúrico, 10\% como ácido salicílico, $10 \%$ como gluorónido fenólico salicílico, 5\% como glucorónidoacílico y $<1 \%$ como ácido gentísico ${ }^{(2)}$.Como se discutió, en sobredosis, la capacidad del hígado para metabolizar la droga se satura y la excreción de salicilato inalterado a nivel renal consiste en una ruta de eliminación importante ${ }^{(4)}$.En dosis usuales, la $t^{1} \frac{2}{2}$ del salicilato en plasma es de 2-3 horas. En casos de intoxicación puede extenderse hasta 15-30 horas $^{(9)}$.

Los cambios en el $\mathrm{pH}$ urinario tienen un efecto significativo en la excreción de salicilatos ${ }^{(15)}$. El aclaramiento es 4 veces mayor a un $\mathrm{pH}$ de 8 que a un $\mathrm{pH}$ de $6^{(2)}$ (mayor que la tasa de filtración glomerular).Si el pH urinario es $>7,5$, más moléculas en la orina se encontraran ionizadas, lo que limita la reabsorción tubular ${ }^{(3)}$.Esto explica porque se puede conseguir un aumento de la excreción al alcalinizar la orina ${ }^{(2 ; 6 ; 10)}$ (ver parte 2).Flujos renales altos disminuyen la reabsorción tubular y lo contrario suceden en oliguria ${ }^{(2)}$.

\section{Normograma de Done}

El conocimiento de la cinética de eliminación del fármaco es esencial para comprender la $\mathrm{t} 1 / 2$ prolongada de los salicilatos en las condiciones de intoxicación y la consecuente duración de las manifestaciones tóxicas ${ }^{(1)}$. El normograma de Done plotea el nivel sérico de salicilato respecto al tiempo de ingestión. El ploteo se realiza 6 horas después de la ingestión y es útil sólo en caso de ingestiones agudas y únicas ${ }^{(16)}$. Sin embargo, a pesar de que fue ampliamente utilizado durante varias décadas, el nomograma de Done no es útil ni adecuado, dado que no considera el cambio de primer orden a orden cero que ocurre cuando los niveles séricos exceden la capacidad de los sistemas de eliminación enzimática ${ }^{(1 ; 4)}$.

\section{Farmacodinamia}

Terapéuticamente, la esterificación del hidroxilo del ácido benzoico confiere a los acetilsalicilatos el potencial de inhibir irreversiblemente la formación de prostanoides ${ }^{(9)}$.La hidrólisis del ácido araquidónico de la membrana plasmática es mediada por la enzima Fosfolipasa $\mathrm{A}_{2}$. A continuación la enzima COX media una reacción de oxigenación y otra de hidroperoxidación, en la que se forma $\mathrm{PGH}_{2}$. Secuencialmente se generan prostanoides de actividad biológica: $\mathrm{PGE}_{2}$, $\mathrm{PGF}_{2 \alpha}, \mathrm{TXA}_{2}$ y $\mathrm{PGI}_{2}$. 
El mecanismo de acción de la AAS depende de la dosis ingerida. A dosis bajas (75-81 mg/día) se presenta una acetilación irreversible de la serina 530 de la $\mathrm{COX}-1$, con lo que se consigue un efecto antitrombótico. A dosis medias (2-4 g/día), se inhibe tanto la COX-1 como la COX-2, asociando efectos antipiréticos y analgésicos. En dosis altas (6-8 g/día) asocian un mecanismo poco conocido y son utilizadas con fines antiinflamatorios, siendo tan eficaces como el cortisol y presentando efectos tanto dependientes como independientes de prostaglandinas, sin embargo el uso de dosis altas está condicionado por su toxicidad $^{(5 ; 12)}$ (Figura 3).

La triada clínica de la intoxicación por salicilatos se manifiesta por: irritación gastrointestinal, tinitus e hiperventilación.Debido a lo inespecífico de los síntomas, la intoxicación temprana puede no ser reconocida. La hiperventilación se puede confundir con agitación emocional o ansiedad, la irritación gastrointestinal puede no estar presente y el tinitus debe ser directamente cuestionado por el médico al paciente. Los signos vitales suelen reflejar un estímulo autonómico aumentado ${ }^{(4)}$.Se debe tener en cuenta además la posibilidad de que el paciente haya ingerido otros fármacos que pueden enmascarar el cuadro y complicar aún más su reconocimiento clínico.

\section{Fisiopatología y manifestaciones clínicas}

Las múltiples alteraciones metabólicas inducidas por el AAS son complejas y no comprendidas totalmente ${ }^{(14)}$. El principal mecanismo fisiopa- tológico de la intoxicación por salicilatos es la interferencia con el metabolismo aerobio, mediante el desacople de la fosforilación oxidativa mitocondrial, por lo que todos los órganos se ven involucrados ${ }^{(4 ; 6)}$. La fisiopatología involucra la inhibición del ciclo del ácido tricarboxílico, inhibición del metabolismo de aminoácidos, estimulación de la glicolisis, gluconeogénesis, catabolismo de grasas y proteínas y estimulación directa e indirecta del centro respiratorio ${ }^{(1)}$.También existe un mecanismo de interacción con el sistema homeostático ${ }^{(1)}$.

Si bien son varios los mecanismos involucrados, se ha descrito que los efectos producidos son dependientes de la concentración plasmática del $\mathrm{AAS}^{(14)}$. Los síntomas de intoxicación se presentan cuando los niveles séricos sobrepasan los 40 $\mathrm{mg} / \mathrm{dL}^{(1)}$.La dosis letal media en adultos es de $10-30 \mathrm{~g}^{(1)}$, es decir 20-60 pastillas de $500 \mathrm{mg}$.De forma general, se ha descrito que la ingesta aguda de $150-200 \mathrm{mg} / \mathrm{kg}$ tiene el potencial de producir una intoxicación leve y una dosis de 200$300 \mathrm{mg} / \mathrm{kg}$ una moderada ${ }^{(7)}$.Mientras que, una dosis de 300-500 mg/kg implica una intoxicación severa $^{(7)}$.Más de $500 \mathrm{mg} / \mathrm{kg}$ representan una toxicidad potencialmente mortal.En dosis tóxicas el ácido salicílico se comporta como un veneno metabólico ${ }^{(4)}$, cuya físiopatología se explicará a continuación

\section{Manifestaciones gastrointestinales}

A nivel de la mucosa gastrointestinal, en el ambiente celular de baja acidez, el ácido débil se ioniza, liberando un hidrogenión que tiende a

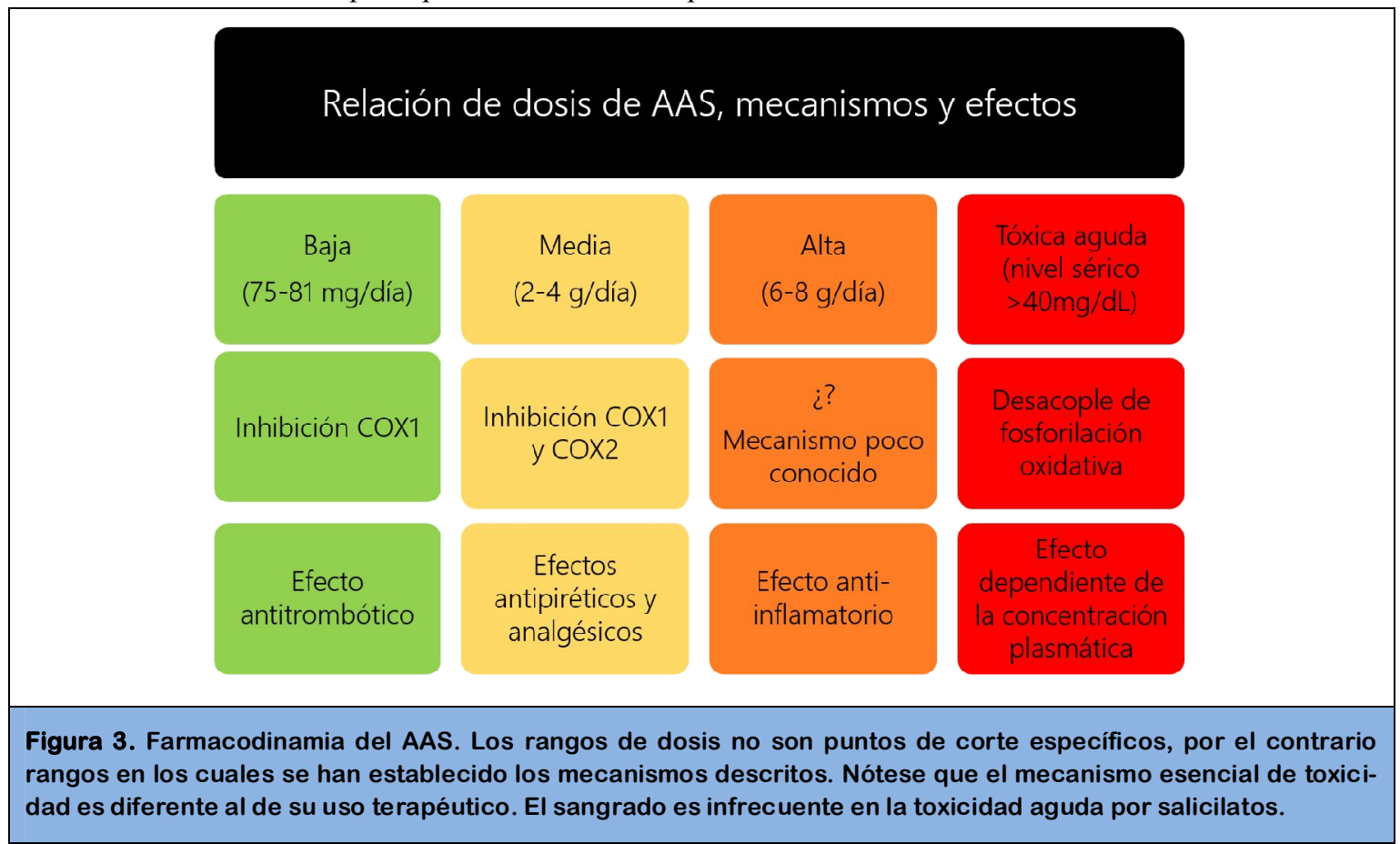


acidificar el ambiente intracelular. En la intoxicación, al entrar una mayor cantidad del AAS, la acidez puede elevarse lo suficiente como para lesionar la célula. Debido a esto es que el cuadro clínico clásico presenta síntomas de irritación gastrointestinal e inclusive en algunos casos sangrado, según el grado de lesión. Comúnmente la sobredosis de AAS se acompaña de dolor abdominal, náuseas y vómitos ${ }^{(3)}$.

Los vómitos prominentes pueden llevar a la depleción de volumen. La hipovolemia reduce la perfusión renal y el flujo urinario, afectado negativamente la eliminación del salicilato y favoreciendo las alteraciones ácido-base y electrolíticas, sobretodo mediante la alcalosis metabóli$\mathrm{ca}^{(3)}$.

\section{Ototoxicidad}

Es un fenómeno bien descrito en el salicilismo, que usualmente se presenta como tinitus, que puede progresar incluso a la sordera (principalmente afectándose los tonos bajos $)^{(1 ; 4)}$. Se ha relacionado el consumo agudo con un efecto de reversibilidad, mientras que el consumo crónico puede presentar afección de manera permanente de las neuronas del ganglio ciliado ${ }^{(13)}$. A pesar de que el mecanismo fisiopatológico no se conoce con certeza, se ha planteado que el efecto es secundario a la interferencia con los canales de cloruro a nivel de las células ciliadas cocleares, que transmiten el sonido ${ }^{(4)}$.Otros mecanismos propuestos son el aumento de la presión laberíntica, bloqueo metabólico de la interfase celular neural, alteración en la actividad gabaérgica mediada por serotonina y vasoconstricción de la microvasculatura auditiva ${ }^{(1 ; 13)}$

\section{Hipertermia}

En el desacople de la cadena respiratoria mitocondrial, la energía que no se transformará ya en ATP se pierde en forma de calor, lo cual aumenta la temperatura corporal.La ausencia de hipertermia no descarta el diagnóstico y puede estar ausente en etapas iniciales ${ }^{(4)}$.

\section{Manifestaciones respiratorias}

El salicilato tiene un efecto de estimulación directa en el centro respiratorio medular a nivel del sistema nervioso central $^{(3)}$. Este mecanismo explica la hiperpnea con que se presenta el paciente. Típicamente la frecuencia respiratoria se encontrará aumentada, sin embargo, el clínico debe observar cuidadosamente el patrón ventilatorio, dado que la frecuencia puede ser normal y estar acompañada de un aumento del volumen tidal $^{(4)}$.

El salicilato también aumenta el metabolismo del músculo esquelético, con lo cual aumenta el consumo de oxígeno y la producción de dióxido de carbono $\left(\mathrm{CO}_{2}\right)$. El aumento en la producción de $\mathrm{CO}_{2}$ constituye también un estímulo respiratorio $^{(3)}$.De forma contraria, concentraciones muy elevadas de salicilato deprimen la respiración, este es un hallazgo tardío en intoxicaciones severas.

También se puede presentar edema pulmo$\operatorname{nar}^{(1 ; 7)}$.Se ha propuesto que el estímulo al sistema nervioso central, produce una liberación masiva de catecolaminas. Esto produce elevación de la presión del atrio izquierdo y retrógradamente de los capilares pulmonares, por lo que típicamente se puede encontrar un paciente con taquicardia y un estado hiperdinámico. Esta condición puede conducir a espasmos neurogénicos de los esfinteres postcapilares, lo que resulta finalmente en edema pulmonar. Además se ha documentado lesión del endotelio pulmonar por el aumento de la permeabilidad vascular ${ }^{(19)}$.La causa del cuadro mencionado anteriormente es no-cardiogénica, la presión vascular pulmonar y la función cardiaca no se encuentran afectadas ${ }^{(3)}$.

\section{Renal}

La presencia de proteinuria de novo suele correlacionarse con una intoxicación seria ${ }^{(6)}$. Se considera que el mecanismo de afección renal es similar al que se presenta a nivel pulmonar (discutido anteriormente) $)^{(3)}$.

\section{Estado ácido-base}

A nivel ácido-base, el paciente inicialmente presentará una alcalosis respiratoria pura, resultado del estímulo respiratorio directo e indirecto ${ }^{(4 ; 6)}$ (discutido previamente).Si el aumento ventilatorio no es capaz de compensar el aumento en la producción de $\mathrm{CO}_{2}$, se desarrollará a la vez acidosis respiratoria ${ }^{(3)}$.La ingestión concomitante de depresores del SNC (e.g. alcohol y benzodiacepinas) puede aumentar el efecto tóxico del salicilato al reducir el estímulo central para la hiperventilación ${ }^{(1)}$.

La alteración mitocondrial conlleva a la interrupción de una serie de funciones enzimáticas y a un 
aumento del metabolismo anaerobio, con conversión celular de piruvato a lactato y el desarrollo rápido de acidosis láctica ${ }^{(4)}$. Debido a la interrupción en la producción de energía a través de la fosforilación oxidativa, también se estimula el catabolismo lipídico y proteico ${ }^{(1)}$.Este mecanismo favorece la producción y acumulación de cuerpos cetónicos ( $\beta$-hidroxibutarato, acetoacetato $\mathrm{y}$ acetona) y de aminoácidos.Además, se presenta una inhibición concomitante de aminotrasferasas, lo cual aumenta aún más la acumulación de aminoácidos ${ }^{(1)}$.

Así, la acidosis metabólica tiene una brecha aniónica aumentada (BA) y se explica por una combinación de lactoacidosis, cetoacidosis $\mathrm{y}$ presencia de aminoácidos, ácido salicílico y $\mathrm{AAS}^{(4 ; 6)}$.La contribución del salicilato por si mismo a la acidosis es poca ${ }^{(1)}$.La literatura menciona casos en los cuales la BA se encuentra normal secundario a pseudohipercloremia ${ }^{(11)}$.

La pérdida de la capacidad compensatoria pulmonar es contraproducente. En un desbalance agudo, los riñones (aún sin patología) son incapaces de compensar suficientemente rápido la $\operatorname{acidosis}^{(3)}$.La alcalemia que se presenta con la alcalosis respiratoria, causa que los riñones aumenten la excreción de bicarbonato y potasio ${ }^{(1)}$. La pérdida urinaria de bicarbonato disminuirá la reserva corporal, lo que complica aún más la compensación del estado de acidosis ${ }^{(1 ; 3)}$.

\section{Homeostasis de la glucosa}

El salicilismo tiene efectos tanto a nivel central como periférico del manejo de la glucosa. Es posible encontrar hiperglicemia debido a que el salicilato aumenta la movilización de las reservas de glicógeno.Pero, también el salicilato es un potente inhibidor de la gluconeogénesis, por lo que se puede presentar hipoglicemia.En estas condiciones la normoglicemia también es una posibilidad $^{(3)}$

\section{Sistema nervioso central}

El mecanismo fisiopatológico de las alteraciones a este nivel es desconocido, sin embargo, clínicamente varía desde confusión hasta estados comatosos. Se ha descrito en animales la presencia de neuroglucopenia a pesar de normoglicemia periférica ${ }^{(3 ; 6)}$.Esto sugiere que la entrega de glucosa a tejidos en el salicilismo es inadecuada, pero se desconoce el mecanismo exacto de este hallazgo. La alteración del estado mental es común en intoxicaciones severas y el desarrollo de edema cerebral puede ser un evento fatal pero infrecuente ${ }^{(6)}$. Se plantea que hay una afección más prominente en casos severos porque habrá una mayor acidosis y el ácido salicílico se encontrará más en su forma no-ionizada, por lo que es capaz de atravesar en mayor mediada la barrera hematoencefálica ${ }^{(1)}$. A parte de los mecanismos ya descritos, se ha propuesto que los salicilatos pueden inducir a nivel cerebral la liberación de factor inductor de apoptosis o del citocromo c, que a su vez son capaces de activar la cascada de las caspasas ${ }^{(18)}$.

\section{Anormalidades hidro-electrolíticas}

Estos cambios son comunes y multifactoriales. El vómito, disminución del aporte oral, diaforesis, hiperventilación, diuresis osmótica (por la presencia de bicarbonato, glucosa y ácidos orgánicos en la orina), aumento del metabolismo e hipertermia, son factores que contribuyen a la pérdida de fluidos y electrolitos.La pérdida de fluidos puede alcanzar los 4-6 L/m ${ }^{2(1)}$.

En la fase alcalótica temprana, la pérdida de bicarbonato condiciona un aumento en la excreción renal de potasio y sodio, como medida para aumentar la reabsorción de hidrogeniones. La diuresis alcalina forzada (ver parte 2) puede también llevar a hipokalemia. La hipernatremia es común y puede deberse a la deshidratación ${ }^{(1)}$.

\section{Anormalidades hematológicas}

Se ha descrito una disminución de la protrombina y factores V, VII y X. Rara vez esta alteración es clínicamente significativa y los sangrados son muy poco frecuentes ${ }^{(1 ; 6)}$.

\section{CONCLUSIONES}

Los salicilatos continúan siendo fármacos ampliamente utilizados en la actualidad. El AAS pertenece a la familia de los ácidos carboxílicos, los cuales presentan importantes funciones terapéuticas analgésicas, antipiréticas, y antitrombóticas. En dosis tóxicas el AAS se comporta como un veneno mitocondrial, ocasionando múltiples perturbaciones a nivel sistémico. La comprensión del comportamiento farmacológico y del proceso fisiopatológico en la intoxicación es 
transcendental para un manejo adecuado y mejor pronóstico del paciente.

\section{BIBLIOGRAFÍA}

1. Kerr F Krenzelok E. Salicylates. En: Shannon $\mathrm{M}$ Borron S Burns $\mathrm{M}$, eds. Haddad and Winchester's Clinical Management of Poisoning and Drug Overdose. Sanders. 4ta edición, 2007:835-848.

2. Grosser T Smyth E Fitzgerald G. Antiinflammatory, antipyretic, and analgesic agents. En: Brunton L Chabner B Knollmann B, eds. Goodman \& Gilman's The Pharmacological Basis of Therapeutics. McGraw-Hill Education. 12va edición, 2010:959-1004.

3. Yip L. Aspirin and salicylates. En: Tintinalli J Stapczynski S Cline D Ma J Cydulka R Meckler $\mathrm{G}$, eds. Tintinalli's emergency medicine: A comprehensive study guide. The American College of Emergency Physicians. 7ma edición, 2010:969

4. O'Malley G. Emergency department management of the salicylate-poisoned patient. Emerg Med Clin North Am. 2007;25(2):333346.

5. Donovan W. Nonsteroidal anti-inflammatory drugs. En: Shannon M Borron S Burns M, eds. Haddad and Winchester's Clinical Management of Poisoning and Drug Overdose. Saunders. 4ta edición, 2007:865-875.

6. Cartwright M Hajja W Al-Khatib S et al. Toxigenic and metabolic causes of ketosis and ketoacidotic syndromes. Crit Care Clin. 2012;28(4):601-631.

7. Cárdenas M. Intoxicación por salicilatos. Imprenta nacional de Colombia. Colombia. 3ra edición, 2009:1262-1265.

8. Kim-Katz S. Salicylates. En: Olson K, eds. Poisoning and drug overdose. McGraw-Hill Professional. 6ta edición, 2011:363-365.

9. Tischendorf N Medina J Pozuelo M Rubio C Hardisson A. Intoxicación por salicilatos. Aula de farmacia. 2009;5):65-75.

10. Prescott L Balali-Mood M Critchley A Johnstone A Proudfoot A. Diuresis or urinary alkalinisation for salicylate poisoning? Bmj. 1982;285(6352):1383-1386.
11. Jacob J Lavonas E. Falsely normal anion gap in severe salicylate poisoning caused by laboratory interference. Ann Emerg Med. 2011;58(3):280-281.

12. Pillinger $\mathrm{M}$ Capodici $\mathrm{C}$ Rosenthal $\mathrm{P}$ et al. Modes of action of aspirin-like drugs: Salicylates inhibit Erk activation and integrin-dependent neutrophil adhesion. Proc Natl Acad Sci. 1998;95(24):14540-14545.

13. Sheppard A Hayes S Chen G Ralli M Salvi R. Review of salicylate-induced hearing loss, neurotoxicity, tinnitus and neuropathophysiology. Acta Otorhinolaryngol Ital. 2014;34(2):79-93.

14. Vale A. Salicylates. Medicine. 2012;40(3):156-157.

15. Vale A. Salicylates. Medicine. 2003;31(10):72-73.

16. Wortzman D Grunfeld A. Delayed absorption following enteric-coated aspirin overdose. Ann Emerg Med. 1987;16(4):434-436.

17. Poisoning with enteric-coated aspirin. Lancet. 1981;2(8238):130.

18. Rauschka H Aboul-Enein F Bauer J Nobis H Lassmann H Schmidbauer M. Acute cerebral white matter damage in lethal salicylate intoxication. NeuroToxicology. 2007;28(1):3337.

19. Yuklyaeva N Chaudhary A Gorantla R Bischof E. Salicylate-induced pulmonary edema- a near-miss diagnosis. Am J Emerg Med. 2014;32(5):495-496.

\section{CONFLICTOS DE INTERES}

Los autores declaran que no existe ningún conflicto de interés. 